\title{
Signal to noise ratio (SNR) comparison for pulsed thermographic data processing methods applied to welding defect detection
}

by P. Albendea *, F. J. Madruga *, A. Cobo * and J. M. López-Higuera *

* Photonic Engineering Group, University of Cantabria, E.T.S.I.I.T. Av. De Los Castros s/n 39005 Santander, Cantabria, Spain; (francisco.madruga, albendeap, miguel.lopezhiguera)@unican.es. Phone / Fax: +34-942200877

\begin{abstract}
In this paper, the use of a signal to noise ratio (SNR) is proposed for the quantification of the goodness of some selected processing techniques of thermographic images, such as skewness and kurtosis based algorithms, the application of the Fourier transform known as Pulsed Phase Transform, or the Principal Component Analysis. They are applied to the quality analysis of different welds of stainless steel plates containing defects such as top and back shielding gases ausence, lack of penetration or perforation in variable thickness plates. The resulting images are compared using the SNR parameter value, which should be higher than $0 \mathrm{~dB}$ if automated detection of defects is required. The performance of each processing technique to detect different defects is analysed.
\end{abstract}

\section{Introduction}

Infrared thermography (IT) applied to the non-destructive testing (NDT) of materials offers a reliable, straightforward and fast means for retrieving structural information from a specimen.

Historically, IT has been applied in industrial environments, for example in the control and monitoring of welding processes in order to guarantee a safe life of the joined parts and to locate different kind of defects. It demands an accurate control of the entire production process, which means the online process monitoring and non-destructive evaluation of final products, and also the inspection of parts in service (offline) so as to detect the priming of damage and monitor its successive growing up. In this context, infrared thermography can be considered as a powerful technique which is able to satisfy both requirements. In the first case, a passive approach (without external excitation) is used. Instead, an active approach, which uses an external excitation source in order to get enough thermal contrast between defective and non defective areas, is necessary for the offline monitoring [1-4].

Specifically, the pulsed active thermography, which is based on the detection of surface temperature anomalies that appear in response to the application of a short thermal pulse (few ms) to the specimen surface, is the most used technique. However, active pulsed thermography presents some limitations fundamentally due to an exponential rate of attenuation of the defect signature with its depth - a consequence of the dependence of thermography on the heat conduction processes to convey information about internal structural anomalies. Defect enhancement techniques must be applied in order to produce a successful thermal inspection. Up to now, found works used some filters as well as the Pulsed Phase Transform (PPT) processing technique, which is based in the Discrete Fourier Transform (DFT), for the inspection of weld seams with different kind of simulated defects like air bubbles, desalignements of the plates, other material inclusions (tungsten), shielding gas ausence or lack of penetration [1, 5-8].

In this paper, PPT [9] and three other specific image processing techniques (skewness [10], kurtosis [11] and PCA [12]), have been applied to pulsed thermographic inspection of welds with different kind of simulated defects in order to improve the thermal inspection, showing both advantages and disadvantages. A technique to quantify the goodness of each processing technique in defect detection using a signal to noise ratio (SNR) is presented. It is applied to TIG welds of stainless steel plates containing defects like top and back shielding gases ausence, discontinuities due to changes in the shielding gas pressure, lack of penetration or perforation due to plates of non constant thickness or inadecuate heat input.

\section{Basic principles of the selected processing techniques}

\subsection{Skewness based algorithm}

Skewness, or the third standardized moment, is a measure of the asymmetry of the probability distribution of a realvalued random variable and it is defined as: 


$$
k_{3}(x)=\frac{E\left[(x-\mu)^{3}\right]}{\sigma^{3}}
$$

where $\mu$ and $\sigma$ are the mean and standard deviation of random variable $x$, respectively, and $E[]$ is the mathematic expectancy defined as

$$
E[X]=\frac{1}{P} \cdot \sum_{n=1}^{P} X_{n}
$$

where $X=\left[\begin{array}{llll}x_{1} & x_{2} & x_{3} & \ldots \\ x_{P}\end{array}\right]$ is a $T x P$ dimensional matrix and $P$ point $x_{n}$ are the $T$-dimension.

A distribution is symmetrical if it shows two identical mirror images when it is split down the middle as the normal distribution (figure 1a). When a distribution is not symmetrical, it has elongated tail at one side (left or right) and there are more data in this side tail that would be expected in a normal distribution. When it happens at the left (figure 1b), the distribution is negatively skewed or skewed to the left, and the in same way, when it happens at the right (figure 1c) the distribution is positively skewed or skewed to the right.

In a typical pulsed thermographic experiment, the evolution of the surface temperature after a heat excitation is recorded into $T$ images ( $n_{x} x n_{y}$ pixels), resulting on a 3D matrix. In order to apply Eq.(1), a preprocessing step is needed to convert the acquired three dimensional data into a two dimensional $T \times P$ data.

The temporal temperature evolution of each pixel of the image sequence obtained after a pulsed thermography testing follows a positively skewed distribution. The skewness parameter calculation can be seen as a process to compress the thermographic sequence into a unique image, which exhibits an improved contrast.

\subsection{Kurtosis based algorithm}

The fourth standardized central moment, or kurtosis, is generally defined as a measure reflecting the degree to which a distribution is peaked. In particular, kurtosis provides information regarding the height of the distribution relative to the value of its standard deviation. Mathematically it is given by:

$$
k_{4}(x)=\frac{E\left\lfloor(x-\mu)^{4}\right\rfloor}{\sigma^{4}}
$$

where $\mu$ and $\sigma$ are, as we defined in the previous headland, the mean and standard deviation of random variable $x$, respectively, and $E[]$ is the mathematic expectancy.

The kurtosis value $(K)$ can be classified in three general categories according to its derivation from a normal distribution: mesokurtic (normal distribution, $K=0$ ), leptokurtic (high degree of peakedness, $K>0$ ) and platykurtic (low degree of peakedness, $K<0$ ). Figure 2 illustrates these three distribution types.

The surface temperature evolution for a non-defective area after pulsed thermography testing follows a leptokurtic distribution, where ambient temperature presents the highest frequency. The kurtosis value in this case is very high. If there is a defect, the distribution will have a bigger or smaller kurtosis value depending on the thermal diffusivity of the defective area material. Using this idea, it is possible to estimate the kurtosis values for every pixel in the image sequence and to obtain a unique image with these values, a kurtogram. The kurtogram will provide an indication of, first, the location of eventual subsurface defects, and second, their thermal diffusivity.

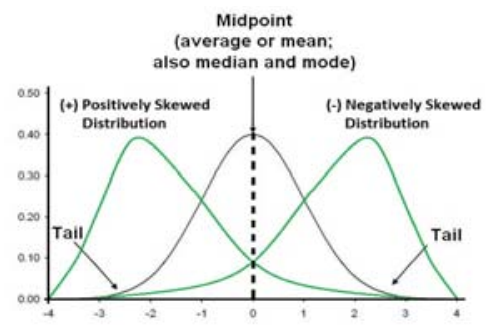

Fig. 1: Example of symmetrical, positively skewed and negatively skewed distributions

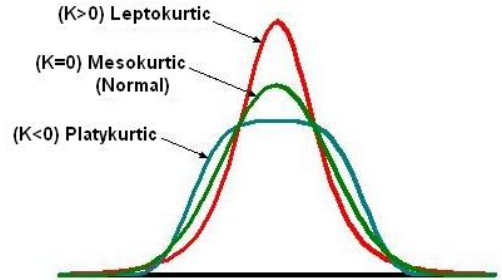

Fig. 2. Example of Leptokurtic, mesokurtic and platykurtic distributions 
As happened with skewness, in order to apply Eq.(3), a preprocessing step is needed to convert the acquired three dimensional data into a two dimensional data.

\subsection{Pulsed Phase Transform (PPT)}

The pulsed phase thermography is a thermographic processing technique that allows a domain change from time to frequency using the discrete Fourier transform (DFT), which is defined as:

$$
F_{n}=\Delta t \cdot \sum_{k=0}^{N-1} T(k \cdot \Delta t) \cdot e^{-i 2 \pi n k / N}=R e_{n}+i \cdot m_{n}
$$

where $n$ is the frequency increment $(n=0,1, \ldots, N), \Delta t$ is the temporal separation between the different acquisitions (images), and $R e_{n}$ and $I m_{n}$ are, respectively, the real and the imaginary parts of the DFT, from which we can obtain the amplitude $\left(A_{n}\right)$ and phase $\left(\Phi_{n}\right)$ components as:

$$
\begin{aligned}
& A_{n}=\sqrt{\operatorname{Re}_{n}^{2}+I m_{n}^{2}} \\
& \phi_{n}=\operatorname{atan}\left(\frac{I m_{n}}{R e_{n}}\right)
\end{aligned}
$$

Considering this, if we have an image sequence with the temporal evolution of the surface temperature of a specimen, we can apply the DFT to the evolution of each pixel in order to get two new sequences with the amplitude and phase images.

\subsection{Principal Component Analysis (PCA)}

As other transformations, e.g. Fourier transform, PCA transforms data into another representation where a new set of basis vectors (variables) are used. In PCA, however, these basis vectors are not constant as is the case in many other transformations. Instead they are calculated based on the data to be transformed. The objective is to enhance the variance of the data set.

PCA is a linear transformation with orthogonal basis vectors, so it can be expressed as translation and rotation:

$$
y=A \cdot\left(x-\mu_{x}\right)
$$

where $A$ contains the new basis vectors, ei as row vectors, hence $A=\left[\begin{array}{llll}e_{1} & e_{2} & \ldots & e_{n}\end{array}\right]^{T}$, and $\mu_{x}$ is the mean of the data set, calculated as follows:

$$
\mu_{x}=\frac{1}{n} \sum_{i=1}^{n} x_{i}
$$

Figure 3 illustrates the basic principles of PCA for the two dimensional case.

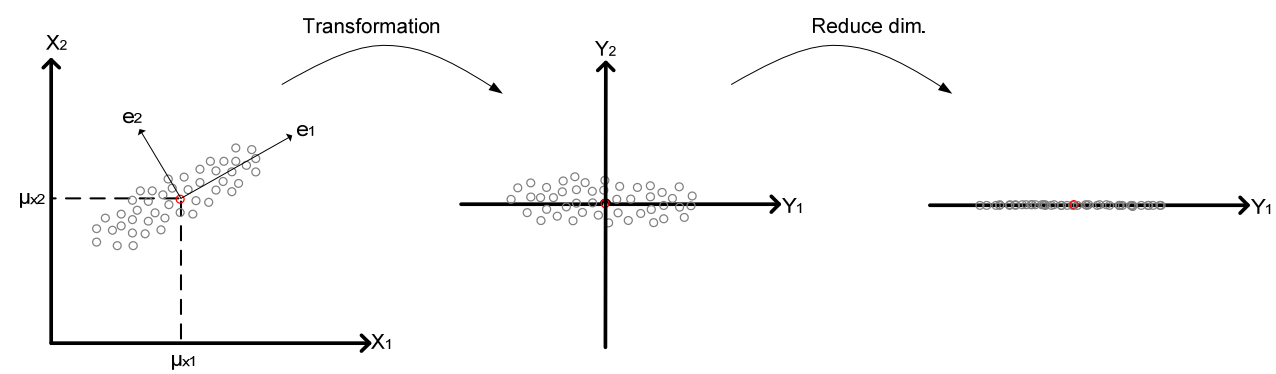

Fig. 3.The basic principle of the PCA 
The first figure illustrates the input where the $i^{\text {th }}$ sample is denoted $x_{i}=\left[\begin{array}{ll}x_{1 i} & x_{2 i}\end{array}\right]^{T}$. The second illustrated the transformed data where the $i^{\text {th }}$ sample is denoted $y_{i}=\left[y_{1 i} y_{2 i}\right]^{T}$ and calculated using the Eq.(6). We can observe that the main part of the data variance is represented in the first variable, $y_{1}$. Therefore, if the second variable is ignored, as is illustrate in the last figure, the main variance of data is kept. In many cases, variance equals information, hence a more compact representation of data/information is obtained.

The process of figuring out which variables to ignore is referred to as the analysis part of the PCA. In that case, we chose three different methods: the so-called "m-method", explained in [12] in the chapter three, the Rajic method [13], and the Marinetti method [14].

In the "m-method", firstly the information of a tridimensional thermographic sequence $V$ of $n_{t}$ images $\left(n_{x} x n_{y}\right.$ pixel) must be organized in a bidimensional one [ $A(m \times n$ pixel)], and it could be done in different ways (see figure 4$)$, depending on the desired reduction (Case 1: number de images in the time, or Case 2: number of pixels of each image):

Case 1: $A_{1}$ has $m=n_{x} \times n_{y}$ rows and $n=n_{t}$ columns (each column is an unrolled image). $\hat{A}_{1}$ is the mean image.

Case 2: $A_{2}$ has $m=n_{t}$ rows (each row is an unrolled image) and $n=n_{x} \times n_{y}$ columns. $\hat{A}_{2}$ is the mean temporal profile. Is pleased that $A_{1}=A_{2}{ }^{T}$.

After that the mean value is substracted $(\hat{A}=A-\bar{A})$ and the covariance matrix calculated. Then the eigenvalues and eigenvectors of the covariance matrix are obtained, and finally the new dataset is derived from the choosing components.

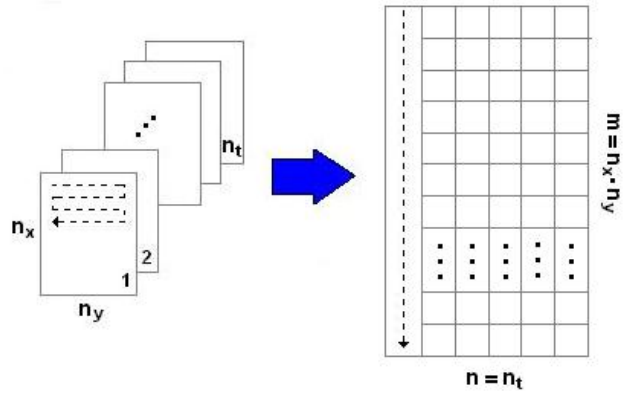

(a)

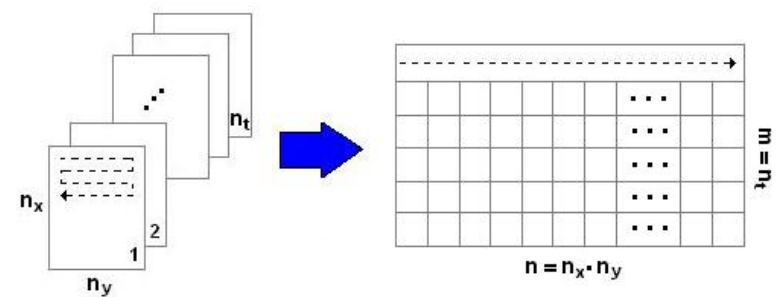

(b)

Fig. 4. Matrix reconstructions in PCA analysis for reduce (a) the number of images and (b) the number of pixels of each image.

Rajic uses case 1 and applies a variation of the m-method in the media substraction, dividing by the standard deviation in order to guarantee a uniform variance in all the images. It is known as standard normalization:

$$
\hat{A}=\frac{A-\bar{A}}{\sigma}
$$

Marinetti chooses the case 2 because the scatter matrix $S$ ( $m \times m$ elements) is smaller and it requires less RAM.

\section{Signal to noise ratio (SNR) quantification}

A SNR value [15] is used to determine what processing algorithm is more suitable for certain application. To determine this SNR value, in the resulting image obtained with the processing algorithm two areas are selected: an area inside the defect and an area around it (sound area), as is showed in figure 5. For each area the medium value is calculated.

In order to detect the defects, it is necessary to have enough thermal contrast between defective and non-defective areas. Because of that, the defect area is considered as "signal" (Sarea) and the sound area as "noise" (Narea) so that the SNR is calculated as is showed in Ec.9, where $\sigma$ is the standard deviation of the noise: 


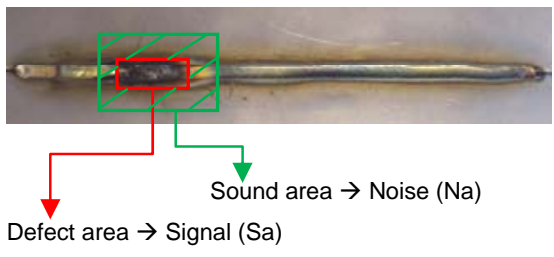

$\mathrm{SNR}_{1}=\frac{\mathrm{S}}{\mathrm{N}}=20 \cdot \log _{10}\left(\frac{\text { abs }\left(\text { Sarea }_{\text {Average }}-\text { Narea }_{\text {average }}\right)}{\sigma}\right) \quad[\mathrm{dB}]$

Fig. 5: Area selection for SNR calculation

For each defect, it is important to observe if it is more or less diffusive than its environment. In the first case the defect temperature (or IR radiation) is higher than in the sound area, and the opposite occurs in the second one. The proposed method for calculating the S/N ratio, which is defined to measure the quality of image constrast for TV transmision systems, is independent of how the defects appear, that is, the defect could be cooler or heater than the surroundings and the result don't change because the absolute value is calculated.

\section{Experimental setup and results}

Different TIG welds of stainless steel plates containing divers kind of defects have been analized by pulsed thermography. The setup used in the weldings creation is shown in figure $6 \mathrm{a}$. The welding system is formed by a "Kemppi Mastertig 2200" power source, a welding torch that remains fixed and a high-precision positioning system with two linear stages (Newport MTM100PP1) of $1 \mathrm{~mm}$ resolution. The AISI-304 stainless steel plates (1mm thickness) used in the experiments were fastened to the positioning system, controlled by a Newport MM4005 controller. The configuration of the positioning system allows to perform weld seams in the directions defined by the two linear stages. The positioning system and tungsten rod electrodes ( $1 \mathrm{~mm}$ diameter) were used, placing the end of the electrode at $2 \mathrm{~mm}$ from the plates, and argon was used as shielding gas, with a typical and constant flow rate of $12 \mathrm{~L} / \mathrm{min}$.

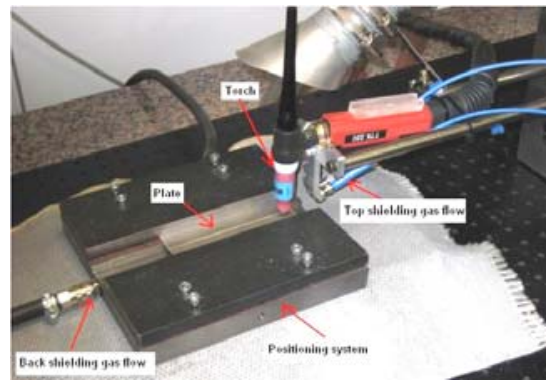

(a)

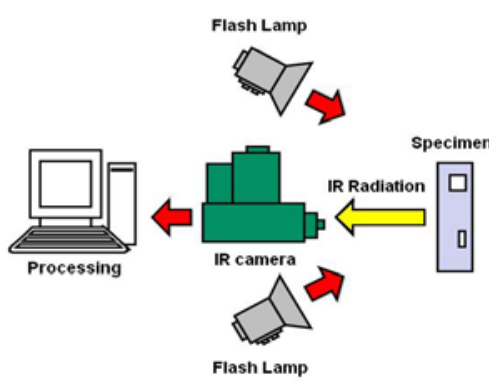

(b)

Fig. 6. Schematic description of the experimental setup used (a) to perform the TIG welds and (b) to record the thermographic image sequences.

Two flash lamps of $75 \mathrm{~W}$ and 1/600s pulse duration have been used to excite the specimen in reflection mode. The surface heating proccess was recorded with a medium wave IR camera (model CMT 128 SM of Thermosensorik) configured to capture 600 frames per second and with 128x128 pixels resolution. As result 1500 image sequences were obtained. The employed experimental setup is shown schematically in figure $6 \mathrm{~b}$.

The obtained thermographic sequences were processed with all the mencioned algorithms. The resulting images are provided with the corresponding SNR value for each kind of defect. For PPT technique, phase images (Eq.(5.2)) have been selected.

The analyzed stainless steel weldings have diferent kind of simulated defects, such as top and back shielding gases ausence, and lack of penetration or perforation due to plates of non constant thickness or inadecuate heat input. The description and number of defects of each weld seam is explained in table1. Both front and back images of each welding are presented. Last ones are inverted with respect the vertical in order to facilitate the identification of defects in the thermographic images.

The resulting images for each welding are shown in figures 7 to11, and the corresponding SNR values in table 2 . Results show that skewness based algorithm allows the automated detection of almost all kind of defects (because SNR values are higher than $\mathrm{OdB}$ ), excluding shortage of both top and back shielding gases flows and perforation due to plates of non constant thickness. The best results are obtained for the detection of discontinuities due to changes in the back shielding gas flow pressure and perforation with sagging due to excessive heat input. In the last case it is the most appropiate 
technique (SNR=28,2094). Kurtosis, however, only allows the detection perforation with sagging $(S N R=16,0938)$, and sometimes detects discontinuities, but provides very bad results in the other cases.

Table 1. Kind and amount of induced defects

\begin{tabular}{|c|c|c|c|}
\hline $\begin{array}{c}\text { WELD } \\
\#\end{array}$ & FRONT \& BACK & $\begin{array}{l}\text { Number of } \\
\text { defects }\end{array}$ & KIND OF DEFECT \\
\hline 1 & $-\infty+\infty=$ & 2 & $\begin{array}{l}\text { Front: shortage of the top } \\
\text { shielding gas flow; Back: } \\
\text { shortage of the back shielding } \\
\text { gas flow }\end{array}$ \\
\hline 2 & 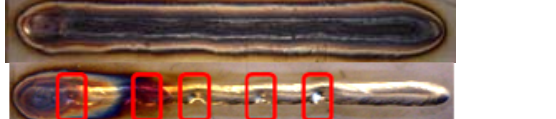 & 5 & $\begin{array}{l}\text { Back: discontinuities due to } \\
\text { changes in the shielding gas } \\
\text { flow pressure }\end{array}$ \\
\hline 3 & $\Longrightarrow$ & 1 & $\begin{array}{l}\text { Lack of penetration due to } \\
\text { inadequate welding }\end{array}$ \\
\hline 4 & $\theta$ & 1 & $\begin{array}{l}\text { Perforations or lack of } \\
\text { penetration due to plates of } \\
\text { non constant thickness }\end{array}$ \\
\hline 5 & 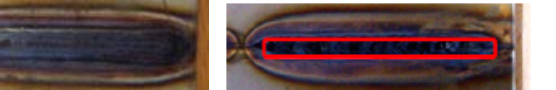 & 1 & $\begin{array}{l}\text { Back: perforation with sagging } \\
\text { due to excessive heat input }\end{array}$ \\
\hline
\end{tabular}

Instead, PPT is the most appropiate technique for the detection of shortage of the back shielding gas flow $(\mathrm{SNR}=3,8580)$ and is also good detecting shortage of the top shielding gas flow, lack of penetration due to inadequate welding, and some discontinuities, but it does not allow the automated detection of perforations and lack of penetration caused by plates of non constant thickness.

Table 2. SNR values for each processing technique and each kind of defect

\begin{tabular}{|c|c|c|c|c|c|c|}
\hline & SKEWNESS & KURTOSIS & PPT & $\begin{array}{c}\text { PCA } \\
\text { m-Method }\end{array}$ & $\begin{array}{c}\text { PCA } \\
\text { Marinetti }\end{array}$ & $\begin{array}{l}\text { PCA } \\
\text { Rajic }\end{array}$ \\
\hline & SNR (dB) & SNR (dB) & SNR (dB) & SNR (dB) & SNR (dB) & SNR (dB) \\
\hline Defects & \multicolumn{6}{|c|}{ WELDING 1} \\
\hline 1 & $-9,8375$ & $-4,0505$ & 8,1458 & 12,0419 & 4,5294 & 1,3689 \\
\hline \multirow[t]{2}{*}{2} & $-9,1385$ & $-8,5821$ & 3,8580 & 1,4479 & $-0,5782$ & 3,2196 \\
\hline & \multicolumn{6}{|c|}{ WELDING 2} \\
\hline 1 & 8,4064 & 7,4520 & 1,0037 & 5,5860 & 6,1653 & 6,1306 \\
\hline 2 & 5,8198 & $-2,4676$ & $-14,0887$ & 4,1930 & 4,7700 & 8,9738 \\
\hline 3 & 0,1182 & $-5,5605$ & $-1,6815$ & 2,9207 & 2,3388 & 1,7165 \\
\hline 4 & $-0,7706$ & $-11,7173$ & 2,2293 & 3,2477 & 0,8421 & $-7,2883$ \\
\hline \multirow[t]{2}{*}{5} & 14,4019 & 7,5727 & $-0,5430$ & 8,3365 & 9,2177 & 9,0731 \\
\hline & \multicolumn{6}{|c|}{ WELDING 3} \\
\hline \multirow[t]{2}{*}{1} & 1,4707 & $-1,4290$ & 5,2371 & $-17,0961$ & 12,3549 & 7,4840 \\
\hline & \multicolumn{6}{|c|}{ WELDING 4} \\
\hline \multirow[t]{2}{*}{1} & $-6,6978$ & $-22,4641$ & $-0,1498$ & $-14,3657$ & $-9,9733$ & $-22,8052$ \\
\hline & \multicolumn{6}{|c|}{ WELDING 5} \\
\hline 1 & 28,2094 & 16,0938 & $-0,2681$ & 9,0090 & 9,4191 & 6,5005 \\
\hline
\end{tabular}

PCA based in m-method is one of the best procesing techniques, because enables the automated detection of almost all kind of defects. It is specially good at detecting discontinuities due to changes in the back shielding gas flow pressure and perforations, and is the best detecting shortage of the top shielding gas flow (SNR=12,0419), although lack of penetration due to both inadequate welding and plates of non constant thikness is not detected. The method applied by Marinetti provides also good results in the detection of discontinuities, perforation with sagging and shortage of the top 
shielding gas flow, and there is not a better technique in the detection of lack of penetration due to inadecuate welding $(\mathrm{SNR}=12,3549)$. Finally, PCA based in the method used by Rajic allows the automatic detection of shortage of both top and back shielding gases flows, discontinuities, lack of penetration and perforations with sagging, but it's not the best in any case. Only perforations due to plates of non constant thikness are not detected (SNR=-22.8052). Because of that it could be considered the best technique.

Considering all these conclusions, if a processing technique must be chosen for the evaluation and the automatic detection of defects in a welding of which nothing is known, PCA based in the method used by Rajic will be the most appropiate technique. However, if the aim is the detection of a particular kind of defect, the chosen algorithm could be another.

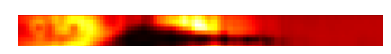

(a) Skewness

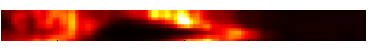

(b) Kurtosis

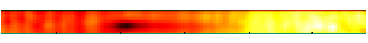

(c) PPT (f=1,2Hz)

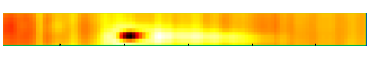

(d) PCA3 m-method

(e) PCA2 Marinetti method

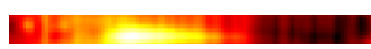

(f) PCA2 Rajic method

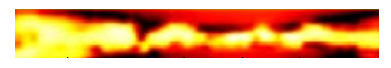

(a) Skewness

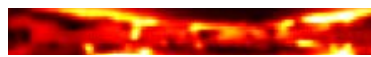

(b) Kurtosis

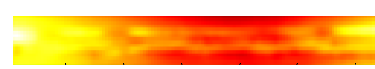

(c) PPT $(f=0,8 \mathrm{~Hz})$

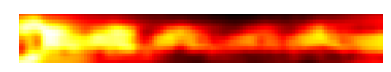

(d) PCA1 m-method

Fig. 7:.Resulting image of each processing technique for welding 1.

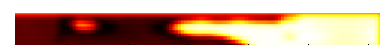

(a) Skewness

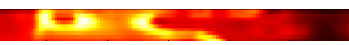

(b) Kurtosis

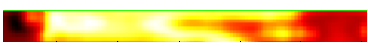

(d) PCA3 m-method

(e) PCA2 Marinetti method

(f) PCA2 Rajic method (c) PPT (f=1,6 Hz)

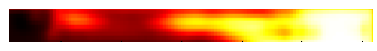

processing technique for

Fig. 9:.Resulting image of each processing technique for
welding 3.

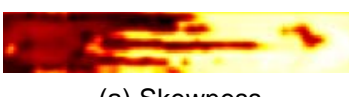

(a) Skewness

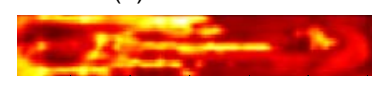

(b) Kurtosis

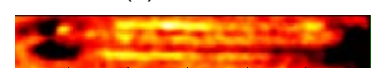

(c) PPT (f=1,6 Hz)
Fig. 8:.Resulting image of each processing technique for welding 2. (e) PCA2 Marinetti method

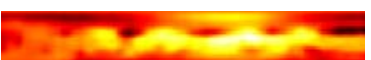

(f) PCA3 Rajic method

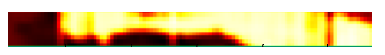

(a) Skewness

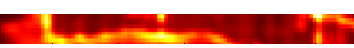

(b) Kurtosis

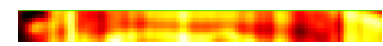

(d) PCA3 m-method

(e) PCA3 Marinetti method

(c) PPT (f=0,8 Hz)

(f) PCA3 Rajic method

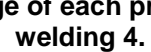

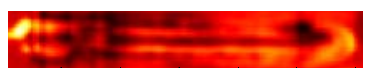

(d) PCA3 m-method

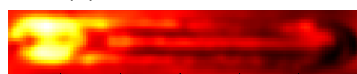

(e) PCA1 Marinetti method

(f) PCA3 Rajic method

Fig. 11:.Resulting image of each processing technique for welding 5.

\section{Conclusions}

Different processing algorithms have been compared using a SNR parameter value. Several TIG welds with different kind of simulated defects have been analyzed and the resulting thermographic sequences have been processed with these algorithms. As result some images were obtained for each welding and for all the defects the SNR value was calculated. That algorithm for which the SNR value is the highest, is the best election for the analysis of this specific kind of defect. The automated detection of defects demands a SNR value higher than one $(0 \mathrm{~dB})$. 
Results show that skewness based algorithm allows the automated detection of almost all kind of defects, excluding shortage of both top and back shielding gases flows and perforation in plates of non constant thickness. It is the most appropiate technique for the detection of perforations with sagging due to excessive heat input. Kurtosis however only allows the detection of perforations and some discontinuities, but is very bad detecting the other kind of defects. PPT is the most appropiate technique for the detection of shortage of the back shielding gas flow but it does not allow the automated detection of perforations due to excessive heat input and plates of non constant thickness.PCA based in m-method achieves the automated detection of almost all kind of defects, being specially good at detecting discontinuities and perforations with sagging, and being the best in the detection of shortage of the top shielding gas flow. The method applied by Marinetti provides also good results and is the best algorithm detecting lack of penetration due to inadequate welding. For the method used by Rajic, it is possible the automatic detection of almost all kind of defects. Only perforations due to plates of non constant thikness are not detected.

\section{ACKNOWLEDGEMENTS}

This work has been co-supported by the Science and Technology Ministry of the Spanish Government through the TEC2007-67987-C02-01 project, and by ENSA, a company from Cantabria (Spain), through two research projects (SISTER1 and TAIS).

\section{REFERENCES}

[1] C. Meola, G. M. Carlomagno, A. Squillace et al., "The use of infrared thermography for nondestructive evaluation of joints," Infrared Physics and Technology, 46(1-2 SPEC. ISS.), 93-99 (2004).

[2] W. Woo, C. W. Chinc, Z. Fenga et al., "Application of infrared imaging for quality inspection in resistance spot welds," Proceedings of SPIE - The International Society for Optical Engineering. Thermosense XXXI, April 14, 2009 - April 15, 2009.7299, The International Society for Optical Engineering (SPIE).

[3] M. Omar, M. Hassan, K. Donohue et al., "Infrared thermography for inspecting the adhesion integrity of plastic welded joints," NDT and E International, 39(1), 1-7 (2006).

[4] U. Siemer, "Simulation and Evaluation of New Thermographic Techniques for the Deployment in the Automotive Industry." 9th European Conference on NDT (ECNDT 2006). September 25th - 29th, 2006.Th.3.4.3, 9 (2006).

[5] N. Nandhitha, N. Manoharan, B. Rani et al., "Detection and Quantification of Tungsten Inclusion in Weld Thermographs for On-line Weld Monitoring by Region Growing and Morphological Image Processing Algorithms." Proceedings of the International Conference on Computational Intelligence and Multimedia Applications, ICCIMA 2007.v.3, 513-518.

[6] J. Medgenberg, and T. Ummenhofer, "Detection of localized fatigue damage in steel by thermography," Proceedings of SPIE - The International Society for Optical Engineering. Thermosense XXIX, April 9, 2007 - April 12, 2007.6541, SPIE.

[7] G. F. Nino, T. J. Ahmed, H. E. N. Bersee et al., "Thermal NDI of resistance welded composite structures," Composites Part B: Engineering, 40(3), 237-248 (2009).

[8] B. Venkatraman, B. Raj, and M. Menaka, "Online infrared detection of inclusions and lack of penetration during welding," Materials Evaluation, 63(9), 933-937 (2005).

[9] C. Ibarra-Castanedo, [Quantitative subsurface defect evaluation by pulsed phase thermography: depth retrieval with the phase] Ph. D. Thesis, Laval University, available online in 2006: http://www. theses. ulaval. ca.

[10] F. Madruga, C. Ibarra-Castanedo, O. Conde et al., "Automatic data processing based on the skewness statistic parameter for subsurface defect detection by active infrared thermography," Proc. QIRT 9-Quantitative Infrared Thermography, (2008).

[11] F. J. Madruga, C. Ibarra-Castanedo, O. M. Conde et al., "Enhanced contrast detection of subsurface defects by pulsed infrared thermography based on the fourth order statistic moment, kurtosis," Proc. SPIE - Int. Soc. Opt. Eng. (USA). Thermosense XXXI, 14 April 2009.7299, 72990 (8 pp.).

[12] T. Moeslund, [Principal component analysis-an introduction] Technical Report CVMT 01-02, ISSN 0906-6233, 2001.

[13] N. Rajic, "Principal component thermography for flaw contrast enhancement and flaw depth characterisation in composite structures," Composite Structures, 58(4), 521-528 (2002).

[14] S. Marinetti, E. Grinzato, P. Bison et al., "Statistical analysis of IR thermographic sequences by PCA," Infrared Physics \& Technology, 46(1-2), 85-91 (2004).

[15] M. A. Omar, and Y. Zhou, "A quantitative review of three flash thermography processing routines," Infrared Physics \& Technology, 51(4), 300-306 (2008). 